\title{
Non-Abelian vortices in Chern-Simons theories and their induced effective theory
}

\author{
L. G. Aldrovandi ${ }^{1,2}$ and F. A. Schaposnik ${ }^{2,3, *}$ \\ ${ }^{1}$ CERN, Theory Division, CH-1211, Geneva 23, Switzerland. \\ ${ }^{2}$ Departamento de Física, Universidad Nacional de La Plata, C.C. 67, (1900) La Plata, Argentina. \\ ${ }^{3}$ CEFIMAS, Santa Fe 1145, C1059ABF - Buenos Aires, Argentina
}

(Received 27 February 2007; published 21 August 2007)

\begin{abstract}
Non-Abelian vortices for a supersymmetric $\mathcal{N}=2$ Chern-Simons-Higgs theory are explicitly constructed. We introduce $N$ Higgs fields in the fundamental representation of the $U(N)$ gauge group in order to have a color-flavor $S U(N)$ group remaining unbroken in the asymmetric phase. Bogomol'nyi-like firstorder equations are found and rotationally symmetric solutions are proposed. These solutions are shown to be truly non-Abelian by parametrizing them in terms of orientational collective coordinates. The lowenergy effective action for the orientational moduli results to be the one-dimensional supersymmetric $\mathcal{N}=2 \mathbf{C P}^{N-1}$ model. We analyze the quantum mechanics of this effective theory in the $N=2$ case.

DOI: 10.1103/PhysRevD.76.045010

PACS numbers: 11.10.Lm, 11.27.+d
\end{abstract}

\section{INTRODUCTION}

Planar physics exhibits many interesting features, both theoretically and experimentally. This is due in part to the fact that the behavior of matter and gauge fields in $d=2$ spatial dimensions can differ in radical ways from the standard behavior we are used to in $d=3$. A clear example of this is the possibility of considering Chern-Simons terms $[1,2]$ whose dynamics is completely different from that of Yang-Mills theories. Chern-Simons gauge theories have been studied extensively in the past, showing several unusual features. For instance, the Abelian theories contain particle excitations carrying fractional spin and statistics, an aspect which has been widely used in many fields in physics, such as the fractional spin in quantum field theory [3-5] and the quantum Hall effect in condensed matter physics [6-9]. It should be noticed also that the high temperature limit of a four-dimensional field theory can be described by a three-dimensional theory where a Chern-Simons term enters naturally. For a nice review, see Ref. [10].

Furthermore, Abelian Chern-Simons-Higgs systems become self-dual when the Higgs potential takes a special sixth-order form so that the energy functional of the systems has a Bogomol'nyi bound [11], which is saturated by the solutions of the self-duality equations [12]. The sixthorder potential has both a symmetric and an asymmetric vacuum. Solutions approaching the asymmetric vacuum at infinity describe topologically stable vortices, whereas solutions which approach the symmetric phase at infinity are nontopological [13]. The same self-dual structure has also been found in $S U(N)$ non-Abelian Chern-Simons-Higgs systems when the Higgs field is in the fundamental representation [14]. The vortices in this case reduce to the Abelian ones, but only stable nontopological solutions are present in both the symmetric and asymmetric phases.

*F. A. S. is associated with CICBA.
Previously, Yang-Mills-Chern-Simons-Higgs theories with the Higgs field in the adjoint representation were investigated for the gauge groups $S U(2)$ [15] and $S U(N)$ [16]. By including $N$ Higgs multiplets in the $S U(N)$ theory (to ensure the maximal breaking of the gauge symmetry), topologically stable non-self-dual vortex solutions were found. These were the first examples known of the socalled $\mathbb{Z}_{N}$ strings, i.e. strings in non-Abelian theories associated with the center of the $S U(N)$ gauge group. Since the models considered contained only a fourth-order potential, these solutions were not found to be self-dual. Self-dual vortex solutions to $S U$ (2) Chern-Simons-Higgs theory with a sixth-order potential were constructed in [17]. However, in these constructions the gauge flux is always directed along a fixed vector in the Cartan subalgebra of $S U(N)$, and no moduli which would make the flux orientation a dynamical variable were found. Therefore, these strings are in this sense Abelian.

Recently, significant progress has been achieved in obtaining genuinely non-Abelian strings in four-dimensional Yang-Mills theories, both $\mathcal{N}=2$ supersymmetric and nonsupersymmetric [18-25]. The bosonic sector of these theories consists basically in a $U(N)$ gauge theory coupled to $N$ Higgs field in the fundamental representation. The resulting models support non-Abelian magnetic flux tubes and non-Abelian confined magnetic monopoles at weak coupling. They are characterized by the presence of orientational moduli associated with the rotation of their color flux in the non-Abelian gauge group $S U(N)$.

It is the purpose of this work to look for non-Abelian string solutions when gauge field dynamics is solely governed by a Chern-Simons action and the symmetry breaking potential is sixth order in order to ensure self-duality and supersymmetric extension. It should be stressed that only in the presence of the Chern-Simons term electrically charged vortices with finite energy (per unit length) do exist $[1,2,26]$ and hence, the model we are interested in could show novel aspects of non-Abelian stringlike configurations. Besides, the existence of some kind of anyons 
with new internal degrees of freedom, as those given by an orientational moduli space, could result in being very useful with respect to their application to condensed matter problems [10].

The paper is organized as follows. We start by considering in Sec. II a supersymmetric $\mathcal{N}=2$ Chern-Simons theory with $U(N)$ gauge group coupled to $N$ scalar multiplets. We obtain for this model the pattern of symmetry breaking and show that in the asymmetric vacuum, the theory lies in the so-called color-flavor locked phase. In Sec. III we begin obtaining the self-duality equations through the usual Bogomol'nyi trick. Next, we find nonAbelian vortices in this model by considering a rotationally symmetric ansatz in the topological sector. These strings are 1/2 BPS saturated and are shown to have a nonAbelian character due to the existence of a set of orientational collective coordinates. Section IV is devoted to finding the low-energy worldline theory describing moduli dynamics which turns out to be the one-dimensional supersymmetric $\mathcal{N}=2 \mathbf{C P}^{N-1}$ model. The quantum mechanics of the effective theory is analyzed in Sec. V for the case $N=2$. A summary and discussion of our results are presented in Sec. VI.

\section{THE THEORY}

Throughout this paper we shall consider the $\mathcal{N}=2$ supersymmetric extension of the $d=2+1$ dimensional non-Abelian Chern-Simons-Higgs theory. The field content of the theory is given by a $U(N)$ vector multiplet consisting in the gauge field $A_{\mu}$, where $\mu, \nu, \ldots=0,1,2$ are Lorentz indices, coupled to scalar multiplets, each of which contain a complex scalar $\phi$ and a Dirac fermion $\chi$. As well as the $U(N)$ gauge symmetry, the Lagrangian also enjoys flavor symmetry. We shall choose the number of flavors $N_{f}=N$. In fact, $N_{f}$ should be larger or equal to the number of colors, $N_{f} \geq N$. Otherwise there will be no nontrivial vacua [19]. The case $N_{f}>N$ which corresponds to semilocal non-Abelian vortices will not be discussed here.

Under these two groups, the scalar multiplets transform as $(\mathbf{N}, \overline{\mathbf{N}})$. Thus, $\phi$ and $\chi$ fields can be seen as $N \times N$ matrices $\phi=\phi^{i}{ }_{a}$ and $\chi=\chi_{a}^{i}$, where the indices $i, j, \ldots=1,2, \ldots, N$ refer to the gauge group and $a, b, \ldots=1,2, \ldots, N$ to the flavor group.

We represent the gauge fields in terms of matrices in the fundamental representation of $U(N)$, that is, $A_{\mu}=A_{\mu}^{r} T^{r}$, where $T^{r}\left(r, s, \ldots=1,2, \ldots, N^{2}\right)$ are the generators of the $\mathbf{N}$ representation. We take the space-time metric $\eta_{\mu \nu}=$ $\operatorname{diag}(+1,-1,-1), \epsilon^{012}=+1$ and use anti-Hermitian generators $T^{r}$ satisfying

$$
\begin{gathered}
{\left[\left(T^{r}\right)^{i}{ }_{j}\right]^{*}=-\left(T^{r}\right)^{j}{ }_{i}, \quad\left[T^{r}, T^{s}\right]=f^{r s t} T^{t},} \\
\left(T^{r} T^{s}\right)_{i}^{i}=-\frac{1}{2} \delta^{r s} .
\end{gathered}
$$

We shall start writing the $\mathcal{N}=2$ supersymmetric nonAbelian Chern-Simons-Higgs Lagrangian within the superfield formulation. To this end, we accommodate the component fields enumerated above into the supermultiplets: a chiral scalar superfield $\Phi=(\phi, \chi, F)$ and a vector superfield $V=\left(A_{\mu}, \lambda, S, D\right)$. Here $F, \lambda, S$, and $D$ are auxiliary fields. We call $\left(x, \theta_{1}, \theta_{2}\right)$ the coordinates of the $\mathcal{N}=2$ superspace. Following Ref. [27], the non-Abelian supersymmetric Chern-Simons term can be written in the form

$$
\begin{aligned}
\mathcal{L}_{\mathrm{CS}}= & \frac{i}{2} \kappa \operatorname{tr} \int d^{2} \theta_{1} d^{2} \theta_{2} \int_{0}^{1} d t \bar{D}^{\alpha}\left(\exp (V(t)) D_{\alpha}\right. \\
& \times \exp (-V)) \exp (V(t)) \partial_{t} \exp (-V(t)) .
\end{aligned}
$$

$V(t)$ is an arbitrary function defined so that

$$
V(t=0)=0, \quad V(t=1)=V .
$$

The integral over the extra parameter $t$ is reminiscent of that arising in the $d=2$ Wess-Zumino-Witten model and can be done explicitly, for general $V(t)$ only after integration on $\theta_{2} . D_{\alpha}$, and $\bar{D}^{\alpha}$ are the usual supercovariant derivatives.

Concerning the matter and the "Fayet-Iliopoulos" Lagrangian, we take

$$
\mathcal{L}_{m}+\mathcal{L}_{\mathrm{FI}}=\int d^{2} \theta_{1} d^{2} \theta_{2}(\operatorname{Tr}(\bar{\Phi} \exp (-V) \Phi)+\operatorname{tr} \xi V) .
$$

Here $\operatorname{Tr}$ represent the trace over color and flavor indexes.

As proven in [27], the complete action

$$
S^{\mathcal{N}=2}=\int d^{3} x\left(\mathcal{L}_{\mathrm{CS}}+\mathcal{L}_{m}+\mathcal{L}_{\mathrm{FI}}\right)
$$

can be written, after integration over $\theta_{2}$, in terms of $\mathcal{N}=$ 1 multiplets, in the form

$$
\begin{aligned}
S^{\mathcal{N}=2}= & -\frac{i \kappa}{4} \operatorname{tr} \int d^{3} x d^{2} \theta_{1}\left(D^{\alpha} \Gamma^{\beta} D_{\beta} \Gamma_{\alpha}\right. \\
& \left.\left.+\frac{1}{3} D_{\alpha} \Gamma_{\beta}\left\{\Gamma^{\alpha}, \Gamma^{\beta}\right\}+\frac{1}{4 !}\left\{\Gamma^{\alpha}, \Gamma^{\beta}\right\} \Gamma_{\alpha}, \Gamma_{\beta}\right\}+B B\right) \\
& +\operatorname{Tr} \int d^{3} x d^{2} \theta_{1}\left(\mathcal{D}^{\alpha} \Phi_{\mid} \overline{\mathcal{D}}_{\alpha} \bar{\Phi}_{\mid}-\frac{i}{2} \bar{\Phi}_{\mid}\left(B+B_{0}\right) \Phi_{\mid}\right. \\
& \left.+\frac{i}{2} \xi B_{0}\right) .
\end{aligned}
$$

Here we have written

$$
V=-i \Gamma^{\alpha} \theta_{2 \alpha}+\frac{i}{2} \theta_{2}^{\alpha} \theta_{2 \alpha}\left(B+B_{0}\right),
$$

where $B$ and $B_{0}$ are auxiliary fields taking values in the Lie algebra of $S U(N)$ and $U(1)$, respectively. The supercovariant derivative $\mathcal{D}^{\alpha}$ is defined as

$$
\mathcal{D}^{\alpha}=D^{\alpha}+\frac{1}{2} \Gamma^{\alpha}, \quad \overline{\mathcal{D}}^{\alpha}=-D^{\alpha}+\frac{1}{2} \Gamma^{\alpha},
$$

and we have denoted 


$$
\Phi_{\mid}=\Phi\left(x, \theta_{1}, \theta_{2}=0\right) .
$$

Once integration over $\theta_{1}$ is performed and the auxiliary fields are eliminated by means of the field equations, the action for the $\mathcal{N}=2$ Chern-Simons-Higgs system takes in component fields the form

$$
\begin{aligned}
\mathcal{L}= & \frac{\kappa}{2} \epsilon^{\mu \nu \rho}\left(F_{\mu \nu}^{r} A_{\rho}^{r}-\frac{e}{3} f^{r s t} A_{\mu}^{r} A_{\nu}^{s} A_{\rho}^{t}\right)+\mathcal{D}_{\mu} \bar{\phi}^{a} \mathcal{D}^{\mu} \phi_{a} \\
& +\frac{i}{2} \bar{\chi}^{a} \mathcal{D}_{\chi_{a}}-\frac{e^{2}}{4 \kappa}\left(\bar{\phi}^{a} T^{r} \phi_{a}-i \xi^{r}\right)\left(\bar{\chi}^{b} T^{r} \chi_{b}\right) \\
& -\frac{e^{2}}{2 \kappa}\left(\bar{\chi}^{a} T^{r} \phi_{a}\right)\left(\bar{\phi}^{b} T^{r} \chi_{b}\right)-\frac{e^{4}}{8 \kappa^{2}}\left(\bar{\phi}^{a}\left\{T^{r}, T^{s}\right\} \phi_{a}\right) \\
& \times\left(\bar{\phi}^{b} T^{r} \phi_{b}-i \xi^{r}\right)\left(\bar{\phi}^{c} T^{s} \phi_{c}-i \xi^{s}\right),
\end{aligned}
$$

where

$$
\begin{gathered}
\bar{\phi}_{i}^{a} \equiv\left(\phi_{a}^{i}\right)^{*}, \quad \bar{\chi}_{i}^{a} \equiv\left(\chi_{a}^{i}\right)^{*}, \\
F_{\mu \nu}^{r}=\partial_{\mu} A_{\nu}^{r}-\partial_{\nu} A_{\mu}^{r}+e f^{r s t} A_{\mu}^{s} A_{\nu}^{t},
\end{gathered}
$$

and

$$
\begin{aligned}
\mathcal{D}_{\mu} \phi^{i}{ }_{a} & \equiv \partial_{\mu} \phi^{i}{ }_{a}+e A_{\mu}^{r}\left(T^{r}\right)^{i}{ }_{j} \phi^{j}{ }_{a}, \\
\mathcal{D}_{\mu} \bar{\phi}^{a}{ }_{i} & \equiv \partial_{\mu} \bar{\phi}^{a}{ }_{i}-e A_{\mu}^{r}\left(T^{r}\right)^{j}{ }_{i} \bar{\phi}^{a}{ }_{j}, \\
\mathcal{D}_{\chi^{i}{ }_{a}} & \equiv \not \chi{ }^{i}{ }_{a}+e \not A^{r}\left(T^{r}\right)^{i}{ }_{j} \chi^{j}{ }_{a} .
\end{aligned}
$$

For simplicity we do not write gauge group indices which are summed, e.g.,

$$
\begin{aligned}
\left(\bar{\phi}^{a} T^{r} \phi_{a}\right) & \equiv\left(T^{r}\right)_{j}^{i} \bar{\phi}_{i}^{a} \phi^{j}{ }_{a}, \\
\left(\bar{\chi}^{a} T^{r} \chi_{a}\right) & \equiv\left(T^{r}\right)_{j}^{i} \bar{\chi}^{a}{ }_{i} \chi^{j}{ }_{a}, \quad \text { etc. }
\end{aligned}
$$

Besides, all the barred spinors will denote complex conjugates of the unbarred ones (see the appendix for our spinor conventions).

The Fayet-Iliopoulos parameters $\xi^{r}$ are nonvanishing only for the $U(1)$ factor group in the total gauge group (we take $\xi^{r=1} \equiv \sqrt{N / 2} \xi$ ). As usual for non-Abelian Chern-Simons theories, a consistent (gauge invariant) quantization requires the coefficient $\kappa$ to be [1]

$$
\kappa=\frac{m e^{2}}{8 \pi} \quad(m= \pm 1, \pm 2, \ldots) .
$$

Note that the renormalizable sixth-order potential for the scalar field is not related to the superpotential (which in our model is absent) and it is uniquely determined by the $\mathcal{N}=$ 2 supersymmetry of the model. Lagrangian (10) coincides with that proposed in Refs. [28,29].

The equations of motion obtained from the variation of the action are

$$
\begin{aligned}
\mathcal{D}_{\mu} \mathcal{D}^{\mu} \phi_{a}= & -\frac{e^{2}}{4 \kappa} T^{r} \phi_{a}\left(\bar{\chi}^{b} T^{r} \chi_{b}\right) \\
& +\frac{e^{2}}{2 \kappa} T^{r} \chi_{a}\left(\bar{\chi}^{b} T^{r} \phi_{b}\right) \\
& -\frac{e^{4}}{8 \kappa^{2}}\left\{T^{r}, T^{s}\right\} \phi_{a}\left(\bar{\phi}^{b} T^{r} \phi_{b}-i \xi^{r}\right) \\
& \times\left(\bar{\phi}^{c} T^{s} \phi_{c}-i \xi^{s}\right) \\
& -\frac{e^{4}}{4 \kappa^{2}} T^{r} \phi_{a}\left(\bar{\phi}^{b}\left\{T^{r}, T^{s}\right\} \phi_{b}\right)\left(\bar{\phi}^{c} T^{s} \phi_{c}-i \xi^{s}\right), \\
i \mathcal{D}_{\chi_{a}}=\frac{e^{2}}{2 \kappa} & T^{r} \chi_{a}\left(\bar{\phi}^{b} T^{r} \phi_{b}-i \xi^{r}\right)+\frac{e^{2}}{\kappa} T^{r} \phi_{a}\left(\bar{\phi}^{b} T^{r} \chi_{b}\right),
\end{aligned}
$$

$$
\kappa \epsilon^{\mu \nu \rho} F_{\nu \rho}^{r}=e J^{r \mu},
$$

where the conserved matter current $J^{r \mu}=\left(\rho^{r}, \mathbf{J}^{r}\right)$ is given by

$$
J^{r \mu}=\mathcal{D}^{\mu} \bar{\phi}^{a} T^{r} \phi_{a}-\bar{\phi}^{a} T^{r} \mathcal{D}^{\mu} \phi_{a}+\frac{i}{2} \bar{\chi}^{a} \gamma^{\mu} T^{r} \chi_{a} .
$$

The 0-component of Eq. (17)

$$
2 \kappa B^{r}=e \rho^{r}
$$

is just the Chern-Simons version of the Gauss law and implies that any object carrying magnetic flux must also carry electric charge.

The $\mathcal{N}=2$ supersymmetry transformation for the Lagrangian (10) are

$$
\begin{aligned}
\delta A_{\mu}^{r} & =\frac{i e}{2 \kappa}\left(\bar{\epsilon} \gamma_{\mu} \bar{\phi}^{a} T^{r} \chi_{a}+\epsilon \gamma_{\mu} \bar{\chi}^{a} T^{r} \phi_{a}\right), \\
\delta \phi_{a} & =\bar{\epsilon} \chi_{a}, \\
\delta \bar{\phi}^{a} & =\epsilon \bar{\chi}^{a}, \\
\delta \chi_{a} & =-2 i \gamma^{\mu} \epsilon \mathcal{D}_{\mu} \phi_{a}+\frac{e^{2}}{\kappa} \epsilon T^{r} \phi_{a}\left(\bar{\phi}^{b} T^{r} \phi_{b}-i \xi^{r}\right), \\
\delta \bar{\chi}^{a} & =-2 i \gamma^{\mu} \bar{\epsilon} \mathcal{D}_{\mu} \bar{\phi}^{a}+\frac{e^{2}}{\kappa} \bar{\epsilon} \bar{\phi}^{a} T^{r}\left(\bar{\phi}^{b} T^{r} \phi_{b}-i \xi^{r}\right) .
\end{aligned}
$$

A supersymmetric vacua of the theory exists whenever the minima of the potential can be set to zero. This happens for constant scalar field configurations satisfying

$$
\begin{aligned}
0 & =\left(\bar{\phi}^{a}{ }_{k}\left(T^{r}\right)^{k}{ }_{l} \phi^{l}{ }_{a}-i \xi^{r}\right)\left(T^{r}\right)_{j}^{i} \phi^{j}{ }_{b} \\
& =-\frac{1}{2}\left(\phi^{i}{ }_{a} \bar{\phi}^{a}{ }_{j}-\xi \delta^{i}{ }_{j}\right) \phi^{j}{ }_{b} .
\end{aligned}
$$

To obtain the last term it is helpful to have the relation satisfied by the matrices of the fundamental representation of $U(N)$,

$$
\left(T^{r}\right)^{i}{ }_{j}\left(T^{r}\right)^{k}{ }_{l}=-\frac{1}{2} \delta_{l}^{i} \delta_{j}^{k}
$$

From Eq. (21) one can see that there are two types of vacua. 
The (gauge) symmetric phase, where

$$
\phi^{i}{ }_{a}=0,
$$

and the asymmetric phase, where

$$
\phi_{a}^{i} \bar{\phi}_{j}^{a}=\xi \delta_{j}^{i} .
$$

It is clear that there is only one vacua in the asymmetric phase which, up to gauge rotations, takes the form

$$
\phi_{a}^{i}=\sqrt{\xi} \delta_{a}^{i} .
$$

The vacuum field (25) has the pattern of symmetry breaking $[18,19]$

$$
U(N)_{\text {color }} \times S U(N)_{\mathrm{flavor}} \rightarrow S U(N)_{\mathrm{c}+\mathrm{f}},
$$

where the surviving unbroken group $S U(N)_{\mathrm{c}+\mathrm{f}}$ is a simultaneous gauge and flavor rotation. Because of this, the theory is said to lie in the color-flavor locked phase.

\section{NON-ABELIAN VORTICES}

\section{A. Bogomol'nyi bound and self-duality equations}

The Hamiltonian for the bosonic sector of the theory is

$$
\begin{aligned}
\mathcal{H}= & \int d^{2} x\left(\mathcal{D}_{0} \bar{\phi}^{a} \mathcal{D}_{0} \phi_{a}+\overrightarrow{\mathcal{D}} \bar{\phi}^{a} \cdot \overrightarrow{\mathcal{D}} \phi_{a}\right. \\
& +\frac{e^{4}}{8 \kappa^{2}}\left(\bar{\phi}^{a}\left\{T^{r}, T^{s}\right\} \phi_{a}\right)\left(\bar{\phi}^{b} T^{r} \phi_{b}-i \xi^{r}\right) \\
& \left.\times\left(\bar{\phi}^{c} T^{s} \phi_{c}-i \xi^{s}\right)\right) .
\end{aligned}
$$

One can find the first-order vortex equations by the usual Bogomol'nyi trick [11]. That is, using the relation

$$
\begin{aligned}
\overrightarrow{\mathcal{D}} \bar{\phi}^{a} \cdot \overrightarrow{\mathcal{D}} \phi_{a}= & \left(\mathcal{D}_{1} \mp i \mathcal{D}_{2}\right) \bar{\phi}^{a}\left(\mathcal{D}_{1} \pm i \mathcal{D}_{2}\right) \phi_{a} \\
& \pm i e B^{r} \bar{\phi}^{a} T^{r} \phi_{a} \mp i \operatorname{tr}\left(\partial_{1} J_{2}^{r}-\partial_{2} J_{1}^{r}\right) T^{r}
\end{aligned}
$$

and the Gauss law Eq. (19), the Hamiltonian can be written as

$$
\begin{aligned}
\mathcal{H}= & \int d^{2} x\left\{\left(\mathcal{D}_{0} \bar{\phi}^{a} \mp \frac{i e^{2}}{2 \kappa}\left(\bar{\phi}^{b} T^{r} \phi_{b}-i \xi^{r}\right) \bar{\phi}^{a} T^{r}\right)\right. \\
& \times\left(\mathcal{D}_{0} \phi_{a} \pm \frac{i e^{2}}{2 \kappa}\left(\bar{\phi}^{c} T^{s} \phi_{c}-i \xi^{s}\right) T^{s} \phi_{a}\right) \\
& \left.+\left(\mathcal{D}_{1} \mp i \mathcal{D}_{2}\right) \bar{\phi}^{a}\left(\mathcal{D}_{1} \pm i \mathcal{D}_{2}\right) \phi_{a}\right\} \pm e \xi \Phi
\end{aligned}
$$

The integral of the last term in the right-hand side of Eq. (28) does not contribute to the energy since it can be written as a surface term vanishing at spatial infinity for any finite-energy solution. On the other hand, $\Phi$ is the topological charge given by

$$
\Phi=-i \int d^{2} x \operatorname{tr} B
$$

which coincides with the $U(1)$ magnetic flux.

The energy is then bounded according to

$$
\mathcal{H} \geq e \xi|\Phi|
$$

It is clear from Eq. (29) that the bound is saturated by configurations satisfying the Gauss law and the self-duality (Bogomol'nyi) equations:

$$
\begin{aligned}
\mathcal{D}_{0} \phi_{a}-\frac{i e^{2}}{2 \kappa} \varepsilon\left(\bar{\phi}^{b} T^{r} \phi_{b}-i \xi^{r}\right) T^{r} \phi_{a} & =0, \\
\left(\mathcal{D}_{1}-i \varepsilon \mathcal{D}_{2}\right) \phi_{a} & =0,
\end{aligned}
$$

with $\varepsilon= \pm$ being the sign of the topological charge. Since static configurations that are stationary points of the energy are also stationary points of the action, the Euler-Lagrange equations of the theory will be satisfied by such static configurations obeying the Gauss law and the self-duality equations.

As it is well known, the existence of a Bogomol'nyi bound for the energy is strongly related to the $\mathcal{N}=2$ supersymmetry of the theory [30]. Indeed, to require that the Bogomol'nyi bound (31) be saturated is equivalent to looking for configurations invariant under half of the supersymmetry transformations (20). In order to verify this, we define the parameters

$$
\epsilon^{ \pm}=\frac{1}{2}\left(1 \pm \varepsilon \gamma^{0}\right) \epsilon
$$

The supersymmetric variation of the fermion field $\chi$ in a bosonic background satisfying Eq. (32) results

$$
\delta \chi_{a}=-2 i\left(\gamma^{\mu} \mathcal{D}_{\mu} \phi_{a}+\varepsilon \mathcal{D}_{0} \phi_{a}\right) \epsilon^{+},
$$

and then the $\chi$ field remains invariant under any supersymmetry transformation with $\epsilon^{+}=0$. Concerning the supersymmetry variation of the bosonic fields, they are automatically zero since we start with a purely bosonic classical configuration. Thus, self-dual solutions are $1 / 2$ BPS, with $\epsilon^{+}$the parameters of the broken supersymmetry and $\epsilon^{-}$the parameters of the unbroken supersymmetry.

\section{B. Non-Abelian multisoliton solution}

In order to find non-Abelian vortices in this model, let us consider rotationally symmetric configurations through the ansatz [19]: 


$$
\begin{aligned}
& \phi=\left(\begin{array}{ccccc}
\varphi(r) & 0 & \cdots & 0 & 0 \\
0 & \varphi(r) & \cdots & 0 & 0 \\
\vdots & \vdots & \ddots & \vdots & \\
0 & 0 & \cdots & \varphi(r) & 0 \\
0 & 0 & \cdots & 0 & e^{i n \theta} \tilde{\varphi}(r)
\end{array}\right) \text {, } \\
& A_{\alpha}^{S U(N)}=\left(\begin{array}{ccccc}
1 & 0 & \cdots & 0 & 0 \\
0 & 1 & \cdots & 0 & 0 \\
\vdots & \vdots & \ddots & \vdots & \vdots \\
0 & 0 & \cdots & 1 & 0 \\
0 & 0 & \cdots & 0 & -(N-1)
\end{array}\right) \\
& \times \frac{i}{N e}\left(\partial_{\alpha} \theta\right)\left[n-f_{N}(r)\right] \text {, } \\
& A_{0}^{S U(N)}=\left(\begin{array}{ccccc}
1 & 0 & \cdots & 0 & 0 \\
0 & 1 & \cdots & 0 & 0 \\
\vdots & \vdots & \ddots & \vdots & \vdots \\
0 & 0 & \cdots & 1 & 0 \\
0 & 0 & \cdots & 0 & -(N-1)
\end{array}\right) i g_{N}(r), \\
& A_{\alpha}^{U(1)}=\sqrt{\frac{2}{N}} \frac{1}{e}\left(\partial_{\alpha} \theta\right)[-n+f(r)], \\
& A_{0}^{U(1)}=\sqrt{2 N} g(r),
\end{aligned}
$$

where $\alpha=1,2$ labels the space coordinates and $(r, \theta)$ are the polar coordinates in this space. Let us note that, as discussed in [19], a more general ansatz corresponding to a charge- $n$ vortex can be obtained just by considering a different partition of $n$ in which each diagonal element of the Higgs field has a winding $\exp \left(i n_{i} \theta\right)$ such that $\sum_{i} n_{i}=n$.

Inserting this ansatz in the Gauss law (19) and the selfduality Eqs. (32), we arrive to the first-order differential equations satisfied by the profile functions

$$
\begin{gathered}
r \partial_{r} \varphi+\frac{\varepsilon}{N}\left(f-f_{N}\right) \varphi=0, \\
r \partial_{r} \tilde{\varphi}+\frac{\varepsilon}{N}\left(f-(1-N) f_{N}\right) \tilde{\varphi}=0, \\
\frac{1}{r} \partial_{r}\left(f-f_{N}\right)-\varepsilon \frac{N e^{4}}{8 \kappa^{2}} \varphi^{2}\left(\varphi^{2}-\xi\right)=0, \\
\frac{1}{r} \partial_{r}\left(f-(1-N) f_{N}\right)-\varepsilon \frac{N e^{4}}{8 \kappa^{2}} \tilde{\varphi}^{2}\left(\tilde{\varphi}^{2}-\xi\right)=0, \\
g_{N}=\frac{\varepsilon e}{4 N \kappa}\left(\tilde{\varphi}^{2}-\varphi^{2}\right), \\
g=-\frac{\varepsilon e}{4 N \kappa}\left(\tilde{\varphi}^{2}-(1-N) \varphi^{2}-N \xi\right) .
\end{gathered}
$$

The boundary conditions at the origin follows from the requirement that the fields be nonsingular. This implies that

$$
n \tilde{\varphi}(0)=0, \quad f_{N}(0)=n, \quad f(0)=n .
$$

At spatial infinity, finiteness of the energy implies that

$$
\begin{aligned}
\varphi(\infty) & =\tilde{\varphi}(\infty)=0 \text { or } \sqrt{\xi}, \\
\varphi(\infty) f(\infty) & =\varphi(\infty) f_{N}(\infty)=0 .
\end{aligned}
$$

In searching for solutions of Eqs. (36), we shall consider only those with $\Phi>0$. Solutions with negative magnetic flux are related to these by the transformation

$$
\begin{aligned}
\varphi \rightarrow \varphi, & \tilde{\varphi} \rightarrow \tilde{\varphi}, & f \rightarrow-f, \\
f_{N} \rightarrow-f_{N}, & g \rightarrow-g, & g_{N} \rightarrow-g_{N} .
\end{aligned}
$$

Note that, with this ansatz, the magnetic flux (30) and the $U(1)$ electric charge take the form

$$
\Phi=\sqrt{\frac{N}{8}} \frac{e}{\kappa} Q^{U(1)}=\frac{2 \pi}{e}(f(0)-f(\infty))=\frac{2 \pi}{e}(n+\alpha),
$$

where we have written $f(\infty) \equiv-\alpha$, being $\alpha=0$ when $\varphi(\infty) \neq 0$ or an undetermined constant when $\varphi(\infty)=0$.

In order to get a better knowledge about these solutions, it will be convenient to define new profile functions $h(r)$ and $\tilde{h}(r)$ as

$$
\begin{aligned}
& h(r)=\frac{1}{N}\left(f(r)-f_{N}(r)\right), \\
& \tilde{h}(r)=\frac{1}{N}\left(f(r)-(1-N) f_{N}(r)\right) .
\end{aligned}
$$

Now, in terms of these functions, Eqs. (36) reduce to two sets of equations, each one being identical to that appearing in the case of the Abelian self-dual Chern-Simons soliton $[12,13]$. That is, a first set of equations for the functions $(\varphi, h)$

$$
r \partial_{r} \varphi+h \varphi=0, \quad \frac{1}{r} \partial_{r} h-\frac{e^{4}}{8 \kappa^{2}} \varphi^{2}\left(\varphi^{2}-\xi\right)=0
$$

with the boundary conditions

$$
h(0)=0, \quad \varphi(\infty)=0 \quad \text { or } \quad \sqrt{\xi}, \quad \varphi(\infty) f(\infty)=0,
$$

and an identical set of equations for the functions $(\tilde{\varphi}, \tilde{h})$

$$
r \partial_{r} \tilde{\varphi}+\tilde{h} \tilde{\varphi}=0, \quad \frac{1}{r} \partial_{r} \tilde{h}-\frac{e^{4}}{8 \kappa^{2}} \tilde{\varphi}^{2}\left(\tilde{\varphi}^{2}-\xi\right)=0,
$$

but with the less restrictive boundary conditions

$$
\begin{gathered}
n \tilde{\varphi}(0)=0, \quad \tilde{h}(0)=n, \quad \tilde{\varphi}(\infty)=0 \quad \text { or } \sqrt{\xi}, \\
\tilde{\varphi}(\infty) \tilde{h}(\infty)=0 .
\end{gathered}
$$

As shown in [12], these equations admit vortexlike solutions for which $\varphi \rightarrow \sqrt{\xi}$ and $\tilde{\varphi} \rightarrow \sqrt{\xi}$ at large distances. They are topologically nontrivial configurations having, as one can see from Eq. (40), quantized magnetic 
flux $\Phi=2 \pi n / e$. Hence, they cannot be continuously deformed to the vacuum solution for topological reasons. There also exist nontopological soliton solutions for which the Higgs field $\phi$ approaches the symmetric minimum at large distances [13]. Their flux is not quantized, but rather it is expressed in terms of the arbitrary parameter $\alpha$ describing the solution. The rest of the paper we will focus on the study of configurations of the former type, that is, solutions in the topological sector. In this case, Eqs. (42) and (44) admit vortexlike solutions for every nonvanishing entire $n$. As we will see, these solutions give truly nonAbelian generalization of the Abelian vortices first discussed in [12].

An important simplification of the ansatz (35) arises as a result of the fact that the vacuum solution,

$$
\varphi(r) \equiv \sqrt{\xi}, \quad h(r) \equiv 0,
$$

is the only solution of Eqs. (42) [13]. Therefore, the Higgs field and the gauge fields in Eq. (35) take the simple form

$$
\begin{aligned}
\phi & =\operatorname{diag}\left(\sqrt{\xi}, \sqrt{\xi}, \ldots, \sqrt{\xi}, e^{i n \theta} \tilde{\varphi}(r)\right) \\
A_{\alpha} & =\operatorname{diag}(0,0, \ldots, 0,1) \frac{i}{e} \partial_{\alpha} \theta(f(r)-n), \quad \alpha=1,2 \\
A_{0} & =\operatorname{diag}(0,0, \ldots, 0,1) \frac{i e}{4 \kappa}\left(\xi-\tilde{\varphi}^{2}\right),
\end{aligned}
$$

where $A_{\mu}=A_{\mu}^{S U(N)}+\frac{i}{\sqrt{2} N} A_{\mu}^{U(1)} I, \mu=0,1,2$. Besides, the profile functions $(\tilde{\varphi}(r), f(r))$ satisfy the following firstorder equations and boundary conditions:

$$
\begin{gathered}
r \partial_{r} \tilde{\varphi}+f \tilde{\varphi}=0, \quad \frac{1}{r} \partial_{r} f-\frac{e^{4}}{8 \kappa^{2}} \tilde{\varphi}^{2}\left(\tilde{\varphi}^{2}-\xi\right)=0, \\
n \tilde{\varphi}(0)=0, \quad f(0)=n, \\
\tilde{\varphi}(\infty)=\sqrt{\xi}, \quad f(\infty)=0,
\end{gathered}
$$

and then, coincide with the profiles of an Abelian self-dual Chern-Simons vortex with $n$ units of topological charge [12].

Let us now discuss some facts about the vortex moduli space. While the vacuum in the asymmetric phase is $S U(N)_{\mathrm{c}+\mathrm{f}}$ symmetric, the solution given by Eq. (35) breaks this symmetry down to $U(1) \times S U(N-1)$. This means that there exists a set of solutions with the same topological charge parameterized by the coset $[18,19]$

$$
\frac{S U(N)_{\mathrm{c}+\mathrm{f}}}{S U(N-1) \times U(1)} \cong \mathbf{C} \mathbf{P}^{N-1}
$$

Thus, in the case of a single unit charge vortex the moduli space decomposes as

$$
\mathcal{M} \cong \mathbf{C} \times \mathbf{C P}^{N-1},
$$

where $\mathbf{C}$ parameterizes the center of mass of the vortex configuration. The presence of these extra orientational collective coordinates makes the vortices genuinely nonAbelian.

Concerning charge- $n$ vortices, one expects from the BPS nature of these configurations that there exist solutions corresponding to $|n|$ well-separated unit charge vortices. Then, as usually happens with solitons satisfying a set of Bogomol'nyi-type equations, the charge- $n$ vortex moduli space should involve as many independent parameters as those necessary to determine the state of $|n|$ independent charge- 1 vortices. We have seen above that each charge- 1 vortex is characterized by its position on the plane together with $(N-1)$ complex parameters describing the orientation of the vortex in the $S U(N)_{\mathrm{c}+\mathrm{f}}$ group. Therefore, the dimension of the charge- $n$ vortex moduli space will be $2|n| N$.

One can make explicit the non-Abelian nature of the solution (35) by applying the color-flavor rotation preserving the asymmetric vacuum. To this end, it is convenient first to pass to the singular gauge where the scalar fields have no winding at infinite, while the vortex flux comes from the vicinity of the origin. Then, the Higgs and the gauge fields can be written as

$$
\begin{aligned}
& \phi=\mathcal{U}\left(\begin{array}{ccccc}
\sqrt{\xi} & 0 & \cdots & 0 & 0 \\
0 & \sqrt{\xi} & \cdots & 0 & 0 \\
\vdots & \vdots & \ddots & \vdots & \vdots \\
0 & 0 & \cdots & \sqrt{\xi} & \\
0 & 0 & \cdots & 0 & \tilde{\varphi}(r)
\end{array}\right) \mathcal{U}^{-1}, \\
& A_{\alpha}^{S U(N)}=-U\left(\begin{array}{ccccc}
1 & 0 & \cdots & 0 & 0 \\
0 & 1 & \cdots & 0 & 0 \\
\vdots & \vdots & \ddots & \vdots & \vdots \\
0 & 0 & \cdots & 1 & 0 \\
0 & 0 & \cdots & 0 & -(N-1)
\end{array}\right) \\
& \times \mathcal{U}^{-1} \frac{i}{N e}\left(\partial_{\alpha} \theta\right) f(r), \\
& A_{0}^{S U(N)}=U\left(\begin{array}{ccccc}
1 & 0 & \cdots & 0 & 0 \\
0 & 1 & \cdots & 0 & 0 \\
\vdots & \vdots & \ddots & \vdots & \vdots \\
0 & 0 & \cdots & 1 & 0 \\
0 & 0 & \cdots & 0 & -(N-1)
\end{array}\right) \\
& \times \mathcal{U}^{-1} \frac{i \varepsilon e}{4 N \kappa}\left(\tilde{\varphi}^{2}-\xi\right), \\
& A_{\alpha}^{U(1)}=\sqrt{\frac{2}{N}} \frac{1}{e}\left(\partial_{\alpha} \theta\right) f(r), \\
& A_{0}^{U(1)}=-\frac{\varepsilon e}{\sqrt{8 N} \kappa}\left(\tilde{\varphi}^{2}-\xi\right),
\end{aligned}
$$

where $\mathcal{U} \in S U(N)$ parameterizes the orientational collective coordinates associated with the flux rotation in $S U(N)$. 


\section{EFFECTIVE VORTEX WORLDLINE THEORY}

In this section we will derive an effective low-energy theory for the orientational collective coordinates on the vortex worldline. Following [23], we parameterize the matrices entering in Eq. (51) as follows:

$$
\begin{aligned}
& \frac{1}{N}\left\{\mathcal{U}\left(\begin{array}{ccccc}
1 & 0 & \cdots & 0 & 0 \\
0 & 1 & \cdots & 0 & 0 \\
\vdots & \vdots & \ddots & \vdots & \vdots \\
0 & 0 & \cdots & 1 & 0 \\
0 & 0 & \cdots & 0 & -(N-1)
\end{array}\right) \mathcal{U}^{-1}\right\}_{j}^{i} \\
& =-n^{i} n_{j}^{*}+\frac{1}{N} \delta_{j}^{i},
\end{aligned}
$$

where $n^{i}$ is a complex vector in the fundamental representation of $S U(N)$, and

$$
n_{i}^{*} n^{i}=1, \quad i=1, \ldots, N .
$$

Note that this gives the correct number of degrees of freedom for the charge- 1 vortex case, namely, $2(N-1)$.

With this parametrization the vortex solution (51) takes the form

$$
\begin{aligned}
\phi & =\frac{1}{N}[\tilde{\varphi}+(N-1) \sqrt{\xi}]+(\tilde{\varphi}-\sqrt{\xi})\left(n \cdot n^{*}-\frac{1}{N}\right), \\
A_{\alpha}^{S U(N)} & =\frac{i}{e}\left(n \cdot n^{*}-\frac{1}{N}\right) \partial_{\alpha} \theta f(r), \\
A_{\alpha}^{U(1)} & =\sqrt{\frac{2}{N}} \frac{1}{e} \partial_{\alpha} \theta f(r), \\
A_{0}^{S U(N)} & =-\frac{i \varepsilon e}{4 \kappa}\left(\tilde{\varphi}^{2}-\xi\right)\left(n \cdot n^{*}-\frac{1}{N}\right), \\
A_{0}^{U(1)} & =\frac{\varepsilon e}{\sqrt{8 N} \kappa}\left(\tilde{\varphi}^{2}-\xi\right),
\end{aligned}
$$

where $\alpha=1,2$. For simplicity we have suppressed all $S U(N)$ indices.

To obtain the low-energy effective theory, we assume that the moduli $n^{i}$ are slowly varying functions of the $x^{0}$ coordinate, promoting the $2(N-1)$ collective coordinates to dynamical fields on the vortex worldline. Plugging the resulting configuration into the Lagrangian (10) and performing the integral over the $\left(x^{1}, x^{2}\right)$ plane, we finish with a one-dimensional sigma model for the bosonic $n^{i}$ coordinates. However, before doing this we have to modify our solution. The point is that our solution was obtained through a color-flavor rotation, which now gets a dependence on the $x^{0}$ coordinate. Therefore, the 0 -component of the gauge potential has to be modified. Following an ansatz similar to that used in [23], we propose

$$
\begin{aligned}
A_{0}^{S U(N)}= & -\frac{i \varepsilon e}{4 \kappa}\left(\tilde{\varphi}^{2}-\xi\right)\left(n \cdot n^{*}-\frac{1}{N}\right) \\
& -\frac{1}{e}\left[\partial_{0} n \cdot n^{*}-n \cdot \partial_{0} n^{*}-2 n \cdot n^{*}\left(n^{*} \partial_{0} n\right)\right] \rho(r), \\
A_{0}^{U(1)}= & \frac{\varepsilon e}{\sqrt{8 N} \kappa}\left(\tilde{\varphi}^{2}-\xi\right),
\end{aligned}
$$

where we have introduced a new profile function $\rho(r)$. It will be determined by its own equation of motion through a minimization procedure.

The kinetic term for $n^{i}$ comes from the gauge and scalar field kinetic terms in Eq. (10), while, due to $n^{i}$ parametrizing the vortex zero modes, no potential term is expected to be present in this sigma model.

Using the latter ansatz for the gauge potential we can calculate the $U(N)$ gauge field strength, which takes the form

$$
\begin{aligned}
F_{0 \alpha}= & -\frac{i}{e}\left(\partial_{0} n \cdot n^{*}+n \cdot \partial_{0} n^{*}\right) \varepsilon_{\alpha \beta} \frac{x^{\beta}}{r^{2}} f(r)(1-\rho(r)) \\
& +\frac{i \varepsilon e}{4 \kappa} \frac{x_{\alpha}}{r} \partial_{r} \tilde{\varphi}^{2}(r) n \cdot n^{*} \\
& +\frac{1}{e}\left[\partial_{0} n \cdot n^{*}-n \cdot \partial_{0} n^{*}-2 n \cdot n^{*}\left(n^{*} \partial_{0} n\right)\right] \\
& \times \frac{x_{\alpha}}{r} \partial_{r} \rho(r)
\end{aligned}
$$

and the other components zero.

Therefore, the Chern-Simons term in the Lagrangian (10) is such that

$$
\epsilon^{\mu \nu \rho}\left(F_{\mu \nu}^{r} A_{\rho}^{r}-\frac{e}{3} f^{r s t} A_{\mu}^{r} A_{\nu}^{s} A_{\rho}^{t}\right)=-\frac{\varepsilon}{\kappa r} f \partial_{r} \tilde{\varphi}^{2} .
$$

Equation (57) implies that when the Chern-Simons governs the gauge field dynamics, the only $x^{0}$-dependent terms contributing to the effective Lagrangian will come from the scalar field kinetic term. After some algebra, one arrives at

$$
S_{\text {eff }}=\frac{2 \pi}{e^{2}} I_{0} \int d x^{0}\left[\partial_{0} n^{*} \partial_{0} n+\left(n^{*} \partial_{0} n\right)^{2}\right],
$$

where the constant $I_{0}$ is given by the following integral defined in terms of the adimensional quantities $\hat{r}=e^{2} r$, $\hat{\varphi}=e^{-1} \tilde{\varphi}$, and $\hat{\xi}=e^{-2} \xi$,

$$
I_{0}=\int_{0}^{\infty} d \hat{r} \hat{r}\left[\left(\hat{\varphi}^{2}+\hat{\xi}\right) \rho^{2}+2(1-\rho)(\hat{\varphi}-\sqrt{\hat{\xi}})^{2}\right]
$$

Minimization of the effective action gives the equation of motion of $\rho$. Note that, contrary to what happens in Yang-Mills theories, in this case $\rho$ results a nondynamical field. Then, it can be put easily in terms of $\tilde{\varphi}$, taking the form

$$
\rho=\frac{(\tilde{\varphi}-\sqrt{\xi})^{2}}{\tilde{\varphi}^{2}+\xi}
$$


Because of action, (58) is invariant under the $U(1)$ gauge transformation

$$
n^{i} \rightarrow e^{i \vartheta(t)} n^{i}, \quad n_{i}^{*} \rightarrow e^{-i \vartheta(t)} n_{i}^{*},
$$

and fields $n^{i}$ are subject to the constraint (53), the effective worldline theory can be recognized as the one-dimensional $\mathbf{C} \mathbf{P}^{N-1}$ theory, as was anticipated already by using symmetry arguments.

\section{QUANTIZATION OF THE ONE-DIMENSIONAL $\mathcal{N}=2 \mathrm{CP}^{1}$ MODEL}

We shall here proceed to the analysis of the model defined by Eq. (58) in the case of $N=2$ both at the classical and quantum levels as a way of describing the low-energy properties of the non-Abelian $U(2)$ ChernSimons vortices. As we have seen in the latter section, the dynamics of the two collective coordinates $n^{1}(t), n^{2}(t)$ is given by a one-dimensional $\mathbf{C P}^{1}$ theory whose Lagrangian takes the form

$$
\mathcal{L}_{B}=\frac{2 \pi}{e^{2}} I_{0}\left[\partial_{t} n^{*} \partial_{t} n+\left(n^{*} \partial_{t} n\right)^{2}\right]
$$

and with the coordinates $n^{1}, n^{2}$ satisfying the constraint

$$
n^{*} n=n_{1}^{*} n^{1}+n_{2}^{*} n^{2}=1 .
$$

In order to quantize this theory, it will be convenient to solve first the constraint Eq. (63) through the stereographic projection and deal with the holomorphic representation of the Lagrangian (62). The holomorphic representation is defined in terms of one complex field $z(t)$ and its complex conjugated $\bar{z}(t)$, which are functions from time onto the Kahler manifold $\mathbf{C P}^{1}$. In this way, the theory describing the dynamics of the bosonic orientational moduli results in an unconstrained one-dimensional $\mathbf{C P}^{1}$ sigma model. Thus, the effective Lagrangian can be written as

$$
\mathcal{L}_{B}=g_{z \bar{z}} \dot{\bar{z}}
$$

where $\dot{z}=\partial_{t} z$ and $g_{z \bar{z}}$ is the $\mathbf{C} \mathbf{P}^{1}$ metric

$$
g_{z \bar{z}}=\frac{r_{0}^{2}}{(1+z \bar{z})^{2}} .
$$

We have renamed the constant coupling as $r_{0}^{2}$, so that $r_{0} \propto$ $e^{-1}$. In the case of $\mathbf{C} \mathbf{P}^{1}$ target space, this implies that the scalar curvature $R$ results in $R \propto e^{2}$. Note that due to the Kahler condition of this manifold, $g_{z \bar{z}}$ can be derived from a Kahler potential, that is, $g_{z \bar{z}}=\partial_{z} \bar{\partial}_{\bar{z}} \mathcal{K}$ with $\mathcal{K}=$ $r_{0}^{2} \log (1+z \bar{z})$.

Moreover, since vortices remain invariant under the action of one half of the supersymmetries, that is, under one complex supercharge, the worldline theory must have $\mathcal{N}=2$ supersymmetries. Thus, we can use the unbroken supersymmetry to reconstruct the fermionic sector of the theory. This implies that the quantum mechanics of the orientational moduli in the low-energy regime is given by a one-dimensional supersymmetric $\mathcal{N}=2 \quad \mathbf{C P}^{1}$ sigma model. The Lagrangian for this theory is

$$
\mathcal{L}=g_{z \bar{z}} \dot{\bar{z}} \dot{\bar{z}}+\frac{i}{4} g_{z \bar{z}}\left(\bar{\psi} \mathcal{D}_{t} \psi-\overline{\mathcal{D}}_{t} \bar{\psi} \psi\right),
$$

where $\psi(t)$ is a complex Grassmann variable and $\bar{\psi}(t)$ its complex conjugated. Besides, $\mathcal{D}_{t}$ and $\overline{\mathcal{D}}_{t}$ are the covariant derivatives

$$
\mathcal{D}_{t} \psi=\dot{\psi}+\Gamma \dot{z} \psi, \quad \overline{\mathcal{D}}_{t} \bar{\psi}=\dot{\bar{\psi}}+\bar{\Gamma} \dot{\bar{z}} \bar{\psi},
$$

with $\Gamma=g^{\bar{z} z} \partial_{z} g_{z \bar{z}}$ and $\bar{\Gamma}=g^{\bar{z} z} \bar{\partial}_{\bar{z}} g_{z \bar{z}}$ being the Christoffel symbols. Lagrangian (66) results invariant under the $\mathcal{N}=$ 2 supersymmetry transformation

$$
\begin{array}{ll}
\delta z=\epsilon \psi, & \delta \psi=-2 i \bar{\epsilon} \dot{z}, \\
\delta \bar{z}=\bar{\psi} \bar{\epsilon}, & \delta \bar{\psi}=2 i \epsilon \dot{\bar{z}} .
\end{array}
$$

In order to proceed to the (canonical) quantization of the theory we find, from (66), the canonical momenta

$$
\begin{array}{rlrl}
p & \equiv \frac{\partial \mathcal{L}}{\partial \dot{z}}=g_{z \bar{z}} \dot{\bar{z}}+\frac{i}{4} g_{z \bar{z}, z} \bar{\psi} \psi, & \pi \equiv \frac{\partial_{l} \mathcal{L}}{\partial \dot{\psi}}=-\frac{i}{4} g_{z \bar{z}} \bar{\psi}, \\
\bar{p} \equiv \frac{\partial \mathcal{L}}{\partial \dot{\bar{z}}}=g_{z \dot{z}} \dot{z}+\frac{i}{4} g_{z \bar{z}, \bar{z}} \psi \bar{\psi}, & \bar{\pi} \equiv \frac{\partial_{l} \mathcal{L}}{\partial \dot{\bar{\psi}}}=-\frac{i}{4} g_{z \bar{z}} \psi,
\end{array}
$$

where $\partial_{l}$ denote the left derivative.

Concerning the Hamiltonian, it can be written as

$$
\mathcal{H} \equiv \dot{z} p+\dot{\bar{z}} \bar{p}+\dot{\psi} \pi+\dot{\bar{\psi}} \bar{\pi}-\mathcal{L}=\dot{z} g_{z \bar{z}} \dot{\bar{z}} .
$$

It follows immediately from (69) that the canonical formalism involves the fermionic second-class constraints

$$
\mathrm{c}^{1}=\pi+\frac{i}{4} g_{z \bar{z}} \bar{\psi}, \quad \mathrm{c}^{2}=\bar{\pi}+\frac{i}{4} g_{z \bar{z}} \psi
$$

This requires use of the formalism of Dirac for constrained systems [31]. Starting from the naïve Poisson brackets

$$
\begin{gathered}
\{z, p\}_{\mathrm{PB}}=1, \quad\{\bar{z}, \bar{p}\}_{\mathrm{PB}}=1, \\
\{\psi, \pi\}_{\mathrm{PB}}=-1, \quad\{\bar{\psi}, \bar{\pi}\}_{\mathrm{PB}}=-1,
\end{gathered}
$$

one defines for any two field variables $f$ and $g$ the Dirac brackets as

$$
\{f, g\}_{\mathrm{DB}} \equiv\{f, g\}_{\mathrm{PB}}-\left\{f, \mathrm{c}^{a}\right\}_{\mathrm{PB}} \mathrm{C}_{a b}^{-1}\left\{\mathrm{c}^{b}, g\right\}_{\mathrm{PB}}, \quad a, b=1,2,
$$

where

$$
\mathrm{C}^{a b} \equiv\left\{\mathrm{c}^{a}, \mathrm{c}^{b}\right\}_{\mathrm{PB}} .
$$

Now, within the Dirac formalism, the basic Dirac brackets result

$$
\{z, p\}_{\mathrm{DB}}=\{\bar{z}, \bar{p}\}_{\mathrm{DB}}=1, \quad\{\psi, \bar{\psi}\}_{\mathrm{DB}}=-2 i g^{\bar{z} z},
$$




$$
\begin{aligned}
& \{\psi, p\}_{\mathrm{DB}}=-\frac{1}{2} \Gamma \psi=\left(\{\bar{\psi}, \bar{p}\}_{\mathrm{DB}}\right)^{*}, \\
& \{\psi, \bar{p}\}_{\mathrm{DB}}=-\frac{1}{2} \bar{\Gamma} \psi=\left(\{\bar{\psi}, p\}_{\mathrm{DB}}\right)^{*},
\end{aligned}
$$

with all other trivial commutation relations omitted.

The $\mathcal{N}=2$ supercharges $Q, \bar{Q}$ corresponding to transformations (68), take the form

$$
Q=p \psi, \quad \bar{Q}=\bar{p} \bar{\psi} .
$$

It is easy to see that these charges satisfy the $\mathcal{N}=2$ superalgebra

$$
\{Q, \bar{Q}\}_{\mathrm{DB}}=-2 i \mathcal{H}, \quad\{Q, Q\}_{\mathrm{DB}}=\{\bar{Q}, \bar{Q}\}_{\mathrm{DB}}=0 .
$$

There is an obvious simpler set of canonical equations, which separate the ordinary and Grassmann variables and which permit the passage to explicit representation of the quantum mechanics. Thus, introducing the tetrad $e, \bar{e}$ given by

$$
e \bar{e}=g \Rightarrow e=\bar{e}=\frac{r_{0}}{1+z \bar{z}},
$$

we define

$$
\lambda=e \psi, \quad \bar{\lambda}=\bar{e} \bar{\psi} .
$$

Therefore, the set $z, p, \lambda$ and its complex conjugate have

$$
\{z, p\}_{\mathrm{DB}}=\{\bar{z}, \bar{p}\}_{\mathrm{DB}}=1, \quad\{\lambda, \bar{\lambda}\}_{\mathrm{DB}}=-2 i
$$

as the only nontrivial canonical equations.

As usual, the passage from the classical theory to the quantum theory is done through the replacement of real variables by Hermitian operators together with the change of brackets

$$
\{,\}_{\mathrm{DB}} \rightarrow-i[\text {, ]. }
$$

It is immediate to find a quantum mechanical representation of the algebra Eq. (82). First, we have for $p$ and $\bar{p}$ the result

$$
p=e^{-1}\left(-i \partial_{z}\right) e, \quad \bar{p}=\bar{e}^{-1}\left(-i \bar{\partial}_{\bar{z}}\right) \bar{e} .
$$

Factors involving the tetrad are required to maintain the Hermiticity relation between $p$ and $\bar{p}$ under the inner product

$$
\left\langle\Psi_{1} \mid \Psi_{2}\right\rangle=\int d^{2} z g_{z \bar{z}} \Psi_{1}^{\dagger}(z, \bar{z}) \Psi_{2}(z, \bar{z}),
$$

where $\Psi(z, \bar{z})$ denotes the "invariant" wave function associated with the ket $|\Psi\rangle$. Second, we can employ a $2 \times 2$ matrix representation for $\psi, \bar{\psi}$, which thus take the form

$$
\psi=\sqrt{2}\left(\begin{array}{ll}
0 & 0 \\
1 & 0
\end{array}\right), \quad \bar{\psi}=\sqrt{2}\left(\begin{array}{ll}
0 & 1 \\
0 & 0
\end{array}\right) .
$$

Concerning the $\mathcal{N}=2$ supercharge $Q$ and $\bar{Q}$, we generalize the classical expression Eq. (78) by adopting the following ordering for the corresponding quantum operators:

$$
\begin{aligned}
Q & \equiv \frac{1}{2}\{p, \psi\}=\psi\left(p-\frac{i}{4} \Gamma\right), \\
\bar{Q} & \equiv \frac{1}{2}\{\bar{p}, \bar{\psi}\}=\bar{\psi}\left(\bar{p}+\frac{i}{4} \bar{\Gamma}\right) .
\end{aligned}
$$

In order to ensure the supersymmetry of the system, we require that the quantum Hamiltonian operator be defined by

$$
\mathcal{H} \equiv \frac{1}{2}\{Q, \bar{Q}\} .
$$

Therefore, using the expressions given for Eqs. (84) and (86), the Hamiltonian takes the form

$$
\mathcal{H}=\left(\begin{array}{cc}
\bar{A} A & 0 \\
0 & A \bar{A}
\end{array}\right)
$$

with

$$
A=i e^{-1}\left(\partial_{z}+\frac{3}{4} \Gamma\right), \quad \bar{A}=i \bar{e}^{-1}\left(\bar{\partial}_{\bar{z}}-\frac{1}{4} \bar{\Gamma}\right) .
$$

As it is well known, eigenfunctions of $\bar{A} A$ and $A \bar{A}$ are closely related due to supersymmetry. More explicitly, if $\varphi_{n}$ is an eigenfunction of $A \bar{A}$ with a nonvanishing eigenvalue $E_{n}$, then $\bar{A} \varphi_{n}$ is an eigenfunction of $A \bar{A}$ with the same eigenvalue $E_{n}$. Concerning the case of vanishing eigenvalue, from the fact that $A^{\dagger}=\bar{A}$ for the inner product (85), one has that zero modes of $A \bar{A}$ and $\bar{A} A$ correspond to states annihilated by $\bar{A}$ and $A$, respectively. It is easy to see the operator $A$ as given by Eq. (90) has no normalizable zero mode, and then neither does the operator $\bar{A} A$. Based on these arguments, we can solve the eigenvalue problem for the Hamiltonian operator $\mathcal{H}$ by considering the following prescription:

$$
\mathcal{H} \Psi_{n}=E_{n} \Psi_{n} \Leftrightarrow \Psi_{n}=\left(\begin{array}{c}
\bar{A} \varphi_{n} \\
\varphi_{n}
\end{array}\right),
$$

where $\left(E_{n}, \varphi_{n}\right)$ solve the eigenvalue problem for the operator $A \bar{A}$, that is, $A \bar{A} \varphi_{n}=E_{n} \varphi_{n}$.

To determine the Hilbert space of eigenstates of the operator $h=A \bar{A}$, let us rewrite it as [32]

$$
\begin{aligned}
h & =-e^{-1} \bar{e}^{-1}\left(\partial_{z}+\frac{\Gamma}{4}\right)\left(\bar{\partial}_{\bar{z}}-\frac{\bar{\Gamma}}{4}\right) \\
& =-\frac{1}{2 r_{0}^{2} \partial_{z} \bar{\partial}_{\bar{z}} \Theta}\left(\partial_{z}-\partial_{z} \Theta\right)\left(\bar{\partial}_{\bar{z}}+\bar{\partial}_{\bar{z}} \Theta\right)
\end{aligned}
$$

with $\Theta=\kappa / 2 r_{0}^{2}=\log \sqrt{1+z \bar{z}}$. Given an operator of the form (92), it is natural to define the "reduced" wave functions $\hat{\varphi}$,

$$
\varphi(z, \bar{z}) \equiv e^{-\Theta} \hat{\varphi}(z, \bar{z})
$$


Thus, the reduced operator $\hat{h}$ becomes

$$
\hat{h}=e^{\Theta} h e^{-\Theta}=-\frac{1}{2 r_{0}^{2} \partial_{z} \bar{\partial}_{\bar{z}} \Theta}\left(\partial_{z}-2 \partial_{z} \Theta\right) \bar{\partial}_{\bar{z}} .
$$

The generator of rotations about the origin in the projected plane $\hat{J}=z \partial_{z}-\bar{z} \bar{\partial}_{\bar{z}}$ commutes with $\hat{h}$. Then, we can diagonalize both simultaneously and decompose $\hat{\varphi}$ as

$$
\hat{\varphi}=z^{j} P(z \bar{z}) .
$$

By writing the undetermined function $P$ as a function of

$$
x \equiv \frac{1-z \bar{z}}{1+z \bar{z}},
$$

the eigenstate condition $\hat{h} \hat{\varphi}=E \hat{\varphi}$ leads to the following ordinary differential equation for the function $P(x)$ :

$$
\left(1-x^{2}\right) \frac{d^{2} P}{d x^{2}}+(1-2 j-3 x) \frac{d P}{d x}+r_{0}^{2} E P=0 .
$$

This is a differential equation of the Jacobi form, whose regular solution in the interval $[-1,1]$ is the Jacobi polynomial

$$
\begin{aligned}
P_{n}^{(j, 1-j)}(x) \equiv & \frac{(-1)^{n}}{2^{n} n !}(1-x)^{-j}(1+x)^{j-1} \frac{d^{n}}{d x^{n}} \\
& \times\left\{(1-x)^{n+j}(1+x)^{n-j+1}\right\} \\
= & \frac{1}{2^{n}} \sum_{m=0}^{n}\left(\begin{array}{c}
n+j \\
m
\end{array}\right)\left(\begin{array}{c}
n-j+1 \\
n-m
\end{array}\right) \\
& \times(1-x)^{n-m}(1+x)^{m} .
\end{aligned}
$$

Note also that the Jacobi polynomial $P_{n}^{(j, 1-j)}$ is defined for $j \geq-n, 1-j \geq-n$, thus leading to upper and lower bounds on the allowed values of $j$.

Putting all these together, we find that the orthonormal set of eigenfunctions of $h=A \bar{A}$ results in

$$
\begin{aligned}
\varphi_{n}^{j}= & \frac{1}{r_{0}} \sqrt{\frac{2 n !(n+1) \Gamma(n+2)}{\pi \Gamma(n+j+1) \Gamma(n-j+2)}} \frac{z^{j}}{\sqrt{1+z \bar{z}}} P_{n}^{(j, 1-j)} \\
& \times\left(\frac{1-z \bar{z}}{1+z \bar{z}}\right), \\
E_{n} & =\frac{1}{r_{0}^{2}} n(n+2), \quad n=0,1,2, \ldots \\
j & =-n,-n+1, \ldots, n+1 .
\end{aligned}
$$

\section{SUMMARY AND DISCUSSION}

One of the goals of this work was to construct and further analyze a new type of stringlike solution in Chern-Simons theories which is genuinely non-Abelian. To do this we adapted recent results obtained in the fourdimensional Yang-Mills theory context, where nonAbelian strings, having many common features with
QCD strings, were founded in Yang-Mills theories. We started by constructing a supersymmetric $\mathcal{N}=2$ ChernSimons theory with $U(N)$ gauge group and coupled to $N$ scalar multiplets. For this theory, we found a Bogomol'nyilike bound for the energy, thus leading to a set of first-order self-duality equations. We solved the self-duality equations by proposing a particular rotationally symmetric ansatz. With this ansatz, equations for the profile functions reduced to those of the Abelian Chern-Simons case. However, thanks to the presence of the color-flavor rotations leaving the asymmetric vacuum invariant, the solutions proposed were found to have a nontrivial orientational moduli space. By applying this color-flavor rotation, we were able to write the gauge and Higgs fields in terms of the orientational collective coordinates.

Another task we accomplished was to obtain the lowenergy theory for the orientational moduli space by assuming slowly varying time dependence for these coordinates and then substituting the resulting configuration in the original Lagrangian. The resulting effective theory corresponds to a one-dimensional $\mathbf{C P}^{N-1}$ sigma model. Since self-dual vortices are 1/2 BPS, the supersymmetric extension of the model corresponds to the $\mathcal{N}=2 \mathbf{C P}^{N-1}$ sigma model. We completed the analysis of the supersymmetric effective theory by quantizing this low-energy theory in the $N=2$ case.

There are several aspects related to our results that deserve future analysis. It particular, one should proceed to a more complete analysis of the multivortex solutions. For this purpose, it would be interesting to look for solutions similar to those found in [33,34], which consist in a composite state of two coincident non-Abelian vortices in Yang-Mills theory. Another relevant topic corresponds to the case of semilocal non-Abelian vortices in ChernSimons theories, expected to appear when the number of flavors is larger than $N$.

Of particular interest is the study of Chern-Simons nonAbelian vortices after quantization, since they could be related to a new type of anyon-like object with possible applications in planar systems. With this in mind, it would be useful to further analyze at the quantum level the lowenergy theories for the orientational moduli. In particular, to extend the $N=2$ results presented here to the $\mathbf{C P}^{N-1}$ model with generic $N$. We should also mention that a particular type of non-Abelian statistic is realized by non-Abelian vortices, which in general arise as solutions of non-Abelian gauge theories with spontaneous symmetry breaking. A family of such solutions was presented in this paper, those in which gauge dynamics is governed by a non-Abelian Chern-Simons theory. Precisely such a gauge theory is known to give an explicit realization of nonAbelian braiding statistics [35] so that our solutions could be of relevance in a condensed matter of contexts as, for example, in connection with the fractional quantum Hall effect $[3-9,36]$. 


\section{ACKNOWLEDGMENTS}

L. A. wishes to thank the Theory Division of CERN for hospitality while part of this work was done. This work was partially supported by PIP6160-CONICET, PICCNRS/CONICET, BID 1728OC/AR, PICT20204ANPCYT grants and by CIC and UNLP.

\section{APPENDIX: CONVENTIONS FOR SPINORS IN $d=\mathbf{2}+\mathbf{1}$}

We use the following representation for the $\gamma$ matrices:

$$
\begin{gathered}
\left(\gamma^{0}\right)_{\alpha}^{\beta}=\left(\sigma^{2}\right)_{\alpha}^{\beta}, \quad\left(\gamma^{1}\right)_{\alpha}^{\beta}=i\left(\sigma^{3}\right)_{\alpha}^{\beta}, \\
\left(\gamma^{2}\right)_{\alpha}^{\beta}=i\left(\sigma^{1}\right)_{\alpha}^{\beta},
\end{gathered}
$$

where $\sigma$ 's are the Pauli's matrices. Then, $\gamma$ matrices satisfy

$$
\left(\gamma^{\mu}\right)_{\alpha}^{\gamma}\left(\gamma^{\nu}\right)_{\gamma}^{\beta}=\eta^{\mu \nu} \delta_{\alpha}^{\beta}-i \epsilon^{\mu \nu \sigma}\left(\gamma_{\sigma}\right)_{\alpha}^{\beta}
$$

The Lorentz rotation generator is realized in the following manner:

$$
\mathcal{M}^{\mu \nu} \equiv \frac{i}{4}\left[\gamma^{\mu}, \gamma^{\nu}\right]=\frac{1}{2} \epsilon^{\mu \nu \rho} \gamma_{\rho} .
$$

Using the representation (A1) one can check the validity of the following relation:

$$
\sigma^{2} \mathcal{M}^{\mu \nu} \sigma^{2}=-\left(\mathcal{M}^{\mu \nu}\right)^{T} .
$$

This gives as a result that $\left(\sigma^{2} \psi\right)^{T} \chi$ is Lorentz invariant. Therefore, we can define the spinor metric as

$$
C^{\alpha \beta} \equiv i\left(\sigma^{2}\right)^{\alpha \beta}=\left(\begin{array}{cc}
0 & 1 \\
-1 & 0
\end{array}\right)=C_{\alpha \beta},
$$

which is used to raise and lower spinor indices via

$$
\theta_{\alpha}=\theta^{\beta} C_{\beta \alpha}, \quad \theta^{\alpha}=C^{\alpha \beta} \theta_{\beta} .
$$

Note that $\gamma$ matrices are anti-Hermitian when lowering their indices, i.e.,

$$
\begin{gathered}
\left(\gamma^{0}\right)_{\alpha \beta}=i \delta_{\alpha \beta}, \quad\left(\gamma^{1}\right)_{\alpha \beta}=i\left(\sigma^{1}\right)_{\alpha \beta}, \\
\left(\gamma^{2}\right)_{\alpha \beta}=-i\left(\sigma^{3}\right)_{\alpha \beta} .
\end{gathered}
$$

Concerning the complex conjugation, we use barred spinors to denote the complex conjugated of the unbarred ones, unlike the usual definition using $\gamma^{0}$ 's. Thus,

$$
\left(\chi^{\alpha}\right)^{*}=\bar{\chi}^{\alpha}, \quad\left(\bar{\chi}^{\alpha}\right)^{*}=\chi^{\alpha} .
$$

Besides, we define the conjugation of a product of spinors as

$$
\left(\chi^{\alpha} \psi^{\beta}\right)^{*}=\bar{\chi}^{\alpha} \bar{\psi}^{\beta}
$$

[1] S. Deser, R. Jackiw, and S. Templeton, Phys. Rev. Lett. 48, 975 (1982).

[2] S. Deser, R. Jackiw, and S. Templeton, Ann. Phys. (N.Y.) 140, 372 (1982); 185, 406(E) (1988); 281, 409 (2000); 281, 409(E) (2000).

[3] J. Frohlich and P. A. Marchetti, Commun. Math. Phys. 121, 177 (1989).

[4] F. Wilczek, Fractional Statistics and Anyon Superconductivity (World Scientific, Singapore, 1990).

[5] A. Lerda, Anyons: Quantum Mechanics of Particles with Fractional Statistics, Lecture Notes in Physics Vol. m14 (Springer, Berlin 1992).

[6] S. C. Zhang, T.H. Hansson, and S. Kivelson, Phys. Rev. Lett. 62, 82 (1989).

[7] J. K. Jain, Phys. Rev. Lett. 63, 199 (1989).

[8] S. C. Zhang, Int. J. Mod. Phys. B 6, 25 (1992).

[9] G. Murthy and R. Shankar, arXiv:cond-mat/9802244.

[10] G. V. Dunne, arXiv:hep-th/9902115.

[11] E. B. Bogomolny, Yad. Fiz. 24, 861 (1976) [Sov. J. Nucl. Phys. 24, 449 (1976)].

[12] J. Hong, Y. Kim, and P. Y. Pac, Phys. Rev. Lett. 64, 2230 (1990); R. Jackiw and E. J. Weinberg, ibid. 64, 2234 (1990).

[13] R. Jackiw, K. M. Lee, and E. J. Weinberg, Phys. Rev. D 42, 3488 (1990).

[14] K. M. Lee, Phys. Rev. Lett. 66, 553 (1991).

[15] H. J. de Vega and F. A. Schaposnik, Phys. Rev. Lett. 56,
2564 (1986).

[16] H. J. de Vega and F. A. Schaposnik, Phys. Rev. D 34, 3206 (1986).

[17] L.F. Cugliandolo, G. Lozano, M. V. Manias, and F. A. Schaposnik, Mod. Phys. Lett. A 6, 479 (1991).

[18] A. Hanany and D. Tong, J. High Energy Phys. 07 (2003) 037.

[19] R. Auzzi, S. Bolognesi, J. Evslin, K. Konishi, and A. Yung, Nucl. Phys. B673, 187 (2003).

[20] M. Shifman and A. Yung, Phys. Rev. D 70, 045004 (2004).

[21] A. Hanany and D. Tong, J. High Energy Phys. 04 (2004) 066.

[22] Y. Isozumi, M. Nitta, K. Ohashi, and N. Sakai, Phys. Rev. D 71, 065018 (2005).

[23] A. Gorsky, M. Shifman, and A. Yung, Phys. Rev. D 71, 045010 (2005).

[24] M. Eto, Y. Isozumi, M. Nitta, K. Ohashi, and N. Sakai, Phys. Rev. Lett. 96, 161601 (2006).

[25] M. Eto, Y. Isozumi, M. Nitta, K. Ohashi, and N. Sakai, J. Phys. A 39, R315 (2006).

[26] B. Julia and A. Zee, Phys. Rev. D 11, 2227 (1975).

[27] E. A. Ivanov, Phys. Lett. B 268, 203 (1991).

[28] H. Nishino and S. J. J. Gates, Int. J. Mod. Phys. A 8, 3371 (1993).

[29] S. J. J. Gates and H. Nishino, Phys. Lett. B 281, 72 (1992).

[30] D. Olive and E. Witten, Phys. Lett. 78B, 97 (1978).

[31] P. A. M. Dirac, Can. J. Math. 2, 129 (1950). 
[32] G. V. Dunne, Ann. Phys. (N.Y.) 215, 233 (1992).

[33] R. Auzzi, M. Shifman, and A. Yung, Phys. Rev. D 73, 105012 (2006).

[34] M. Eto, K. Konishi, G. Marmorini, M. Nitta, K. Ohashi, W. Vinci, and N. Yokoi, Phys. Rev. D 74, 065021 (2006).
[35] E. Verlinde, in Modern Quantum Field Theory (World Scientific, Singapore, 1991).

[36] E. Fradkin, C. Nayak, and K. Schoutens, Nucl. Phys. B546, 711 (1999). 